\title{
Epigenetic Regulation of Chondrocyte Catabolism and Anabolism in Osteoarthritis
}

\author{
Hyeonkyeong Kim, Donghyun Kang, Yongsik Cho, and Jin-Hong Kim*
}

\begin{abstract}
Osteoarthritis (OA) is one of the most prevalent forms of joint disorder, associated with a tremendous socioeconomic burden worldwide. Various non-genetic and lifestyle-related factors such as aging and obesity have been recognized as major risk factors for $O A$, underscoring the potential role for epigenetic regulation in the pathogenesis of the disease. OA-associated epigenetic aberrations have been noted at the level of DNA methylation and histone modification in chondrocytes. These epigenetic regulations are implicated in driving an imbalance between the expression of catabolic and anabolic factors, leading eventually to osteoarthritic cartilage destruction. Cellular senescence and metabolic abnormalities driven by OAassociated risk factors appear to accompany epigenetic drifts in chondrocytes. Notably, molecular events associated with metabolic disorders influence epigenetic regulation in chondrocytes, supporting the notion that $O A$ is a metabolic disease. Here, we review accumulating evidence supporting a role for epigenetics in the regulation of cartilage homeostasis and OA pathogenesis.
\end{abstract}

\section{INTRODUCTION}

$\mathrm{OA}$ is the most prevalent form of arthropathy, the incidence of which increases with age, affecting around $50 \%$ of the aged population (Dillon et al., 2006; Lawrence et al., 2008). OA is primarily characterized by structural damage and functional failure of articular cartilage. Cartilage homeostasis is maintained by chondrocytes, a unique cell type resident in cartilage. Chondrocytes express various extracellular matrix (ECM) molecules such as type II collagen and sulfated proteoglycans, which are essential components of chondrocyte anabolism (Knudson and Knudson, 2001; Lefebvre et al., 1998). Dense aggregates of negatively charged proteoglycans attract water molecules and promote osmotic swelling, endowing the tissue with resistance to compressive loading. A network of crosslinked collagen fibrils, in contrast, provides tensile resistance

Department of Biological Sciences, Seoul National University, Seoul 151-747, Korea

*Correspondence: jinhkim@snu.ac.kr

Received 15 July, 2015; accepted 18 July, 2015; published online 5 August, 2015

Keywords: cartilage, chondrocytes, epigenetics, metabolism, osteoarthritis and counterbalances this osmotic swelling, imparting tissue integrity and load-bearing properties to articular cartilage.

OA chondrocytes, on the other hand, are characterized by accelerated catabolic processes as well as suppression of anabolic processes. Upregulation of matrix-degrading enzymes such as matrix metalloproteinases (MMPs), and a disintegrin and metalloproteinases with thrombospondin motifs (ADAMTS) family drives degradation of cartilage ECM, eventually leading to cartilage destruction (Heinegard and Saxne, 2011; Kim et al., 2014). Inflammatory mediators such as COX2 and NO further contribute to eliciting imbalance between chondrocyte catabolism and anabolism (Houard et al., 2013).

Various etiological factors for OA have been noted, including those unrelated to genetics, such as age, obesity, dietary factors, sedentary life style, and injury (Buckwalter and Brown, 2004; Loeser, 2013; Wluka et al., 2013). However, the precise molecular and cellular mechanisms by which these environmental OA risk factors mediate the disruption of cartilage homeostasis remain elusive. Recent progress in elucidating the epigenetic regulation of chondrocyte catabolism and anabolism has shed light on the molecular pathogenesis of OA.

\section{TYPES OF EPIGENETIC REGULATION IN CHONDROCYTES}

\section{DNA methylation}

DNA methylation, which is associated with the remodeling of chromatin structure, plays an essential role in regulating gene expression. The alteration of chromatin structure induced by DNA methylation blocks the access of transcriptional machineries to the promoter regions of target genes and facilitates the recruitment of repressive chromatin remodeling complexes, collectively causing transcriptional repression (Razin, 1998). DNA methylation patterns are regulated by several different DNA methyltransferases (DNMTs) such as DNMT1, DNMT3A, and DNMT3B (Pradhan and Esteve, 2003). In fact, DNMT1 and DNMT3A are present at high levels in cartilage tissue, suggesting possible roles for these enzymes in the regulation of chondrocyte homeostasis (Sesselmann et al., 2009). However, it was reported that there is no apparent change in the overall DNA methylation content in chondrocytes isolated from OA patients (Sesselmann et al., 2009). Instead, DNA methylation patterns in OA chondrocytes exhibit dynamic alterations when examined at the promoter regions of individual genes (FernandezTajes et al., 2014).

In OA, inflammatory cytokines such as IL-1 $\beta$ promote the expression of matrix-degrading enzymes (Troeberg and Nagase, 
2012). Demethylation of CpG sites within the $I L 1 B$ promoter is required for the expression of this gene in OA-like conditions (Hashimoto et al., 2009; 2013). In addition, the promoters of genes encoding catabolic factors such as MMP3, 9, 13, and ADAMTS4 are demethylated, accounting for the increased expression of these genes under OA-related pathogenic conditions (Roach et al., 2005). Notably, the methylation status of the MMP13 promoter region modulates the access and binding of hypoxia-inducible factor (HIF)-2 $\alpha$ (Hashimoto et al., 2013), which is a master regulator of chondrocyte catabolism (Saito et al., 2010; Yang et al., 2010), adding an extra layer of transcriptional regulation of MMP13. Furthermore, hypomethlyation of the MMP13 promoter exposes the binding region for CAMP response element-binding protein (CREB) and CREB binding protein (CBP), resulting in increased MMP13 expression (Bui et al., 2012).

Similarly, DNA methylation influences the expression levels of anabolic factors during the pathogenesis of OA. Hypermethylation of the COL9A1 enhancer causes transcriptional repression of COL9A1 during OA development by attenuating the binding of SOX9 to the COL9A1 promoter (Imagawa et al., 2014; Zimmermann et al., 2008). In contrast, the expression of COL2A1 and ACAN do not appear to be affected by the methylation status of $\mathrm{CpG}$ sites on their promoters (Imagawa et al., 2014; Poschl et al., 2005; Zimmermann et al., 2008). Alteration of methylation patterns may be accompanied by single nucleotide polymorphisms (SNP), accounting for genetic susceptibility to OA. For instance, there are two SNP sites (rs143383 and rs143384) in the 5'UTR of GDF5, whose roles in joint development and chondroprotection are well-characterized (Reynard et al., 2011). The disease-associated T allele, arising as a result of a SNP (rs143384, C/T) in GDF5 is linked to susceptibility to OA, putatively associated with the aberrant demethylated state of its promoter (Reynard et al., 2011).

\section{Histone modification}

The post-translational modification of histones includes acetylation, methylation, ubiquitination, sumoylation, and phosphorylation. The overall pattern of histone modification regulates interaction between DNA, regulatory proteins, and other histone molecules, thereby affecting gene expression (Kouzarides, 2007).

Histone N-terminal tails protruding from nucleosomes are subjected to acetylation modifications by histone acetyltransferases (HATs), which include three major families: Gcn5 N-acetyltransferases (GNATs), p300/CBP, and MYST family (named for its founder members Morf, Ybf2, Sas2, and Tip60) (Sterner and Berger, 2000). Histone acetylation mediated by p300/CBP leads to transcriptional activation of $S O X 9$, illustrating the role of the HAT family in sustaining chondrocyte homeostasis (Furumatsu and Asahara, 2010; Furumatsu et al., 2005).

Histone deacetylases (HDACs), in contrast, oppose the action of HAT enzymes by removing the acetyl group from lysine residues. HDACs are subdivided into two groups: The SIR2 family of $\mathrm{NAD}^{+}$-dependent protein deacetylases and the classical HDAC family. The SIR2 family, consisting of seven family members in mammals (SIRT1-7), removes acetyl groups from lysine residues of histones $\mathrm{H} 3$ and $\mathrm{H} 4$, and non-histone proteins (Blander and Guarente, 2004; de Ruijter et al., 2003). In chondrocytes, SIRT1, one of the most extensively studied SIRT family members, was shown to promote the expression of cartilage-specific genes in a SOX9-dependent manner (DvirGinzberg et al., 2008). SIRT1 forms a complex with SOX9 and tarqets it for deacetvlation. The complex is subsequently brought to the enhancer and promoter regions of COL2A1,

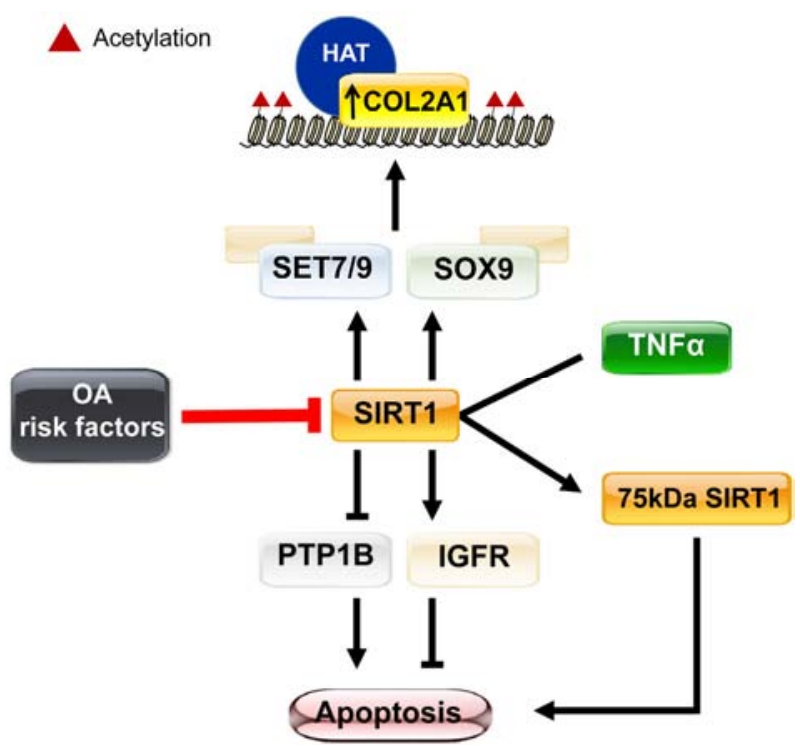

Fig. 1. Epigenetic regulation of chondrocyte anabolism and apoptosis by SIRT1. SIRT1 forms a complex with SET7/9 or SOX9 and recruits HATs, facilitating acetylation of the COL2A1 promoter and expression of COL2A1. SIRT1 inhibits chondrocyte apoptosis by suppressing PTP1B or activating IGF pathway. TNF $\alpha$-mediated generation of 75-kDa SIRT1 inhibits inflammation-induced apoptosis. Downregulation of SIRT1 in OA is responsible for suppression of chondrocyte anabolism and increased apoptosis.

where it further recruits coactivators such as GCN5, PGC1 $\alpha$, and p300, modulating the acetylation state of H3K9/K14 and $\mathrm{H} 4 \mathrm{~K} 5$, and mediating tri-methylation of H3K4 (Dvir-Ginzberg et al., 2008). In line with reports revealing the anabolic roles of SIRT1 in chondrocytes, other chondroprotective roles of SIRT1 have been noted in the context of OA pathogenesis. SIRT1 suppresses protein tyrosine phosphatase 1B (PTP1B) and activates insulin-like growth factor (IGF) receptor pathway, enhancing survival of chondrocytes (Gagarina et al., 2010). Moreover, TNF $\alpha$ signaling triggers cathepsin B-mediated cleavage of SIRT1, producing 75-kDa SIRT1 (Dvir-Ginzberg et al., 2011). This $75-k D a$ SIRT1 has been shown to be implicated in protecting chondrocytes from apoptosis following exposure to proinflammatory cytokines (Oppenheimer et al., 2012). Consistently, heterozygous haploinsufficient Sirt $1^{\text {t/ }}$ or Sirt1 mutant mice lacking SIRT1 enzymatic activity show increased chondrocyte apoptosis and enhanced OA severity with age (Gabay et al., 2012; 2013; Matsuzaki et al., 2014). Furthermore, the expression level of SIRT1 is reduced over the course of OA, giving rise to hypertrophic phenotypes and OA-like gene expression patterns in chondrocytes (Fujita et al., 2011). Similarly, surgically-induced OA pathogenesis in mice was significantly accelerated by chondrocyte-specific deletion of the Sirt1 gene, with concomitant downregulation of anabolic proteins and upregulation of cartilage degrading enzymes (Gabay et al., 2013; Matsuzaki et al., 2014). Moreover, in Sirt1 CKO mice, p65 was found to be acetylated, which in turn causes activation of NF-kB pathway and consequently catabolic pathways, augmenting OA pathogenesis (Fig. 1) (Dvir-Ginzberg et al., 2011). Additionally, SIRT6 has been shown to be responsible for controlling senescence in cartilage by protecting chondrocytes from 
DNA damage, telomere dysfunction, and premature senescence (Nagai et al., 2015).

Classical HDACs remove acetyl groups from N-acetyl lysine residues on histones, enabling the histones to wrap DNA more tightly to repress transcription. The broad inhibition of HDACs by trichostatin A (TSA) or PXD101 was shown to suppress COL2A1 expression in chondrocytes, suggesting that the HDAC family plays a role in regulating chondrocyte anabolism. Moreover, HDAC inhibition promotes acetylation of the WNT-5A promoter, which in turn leads to transcriptional suppression of COL2A1 (Huh et al., 2007). HDAC1 and HDAC2 activity were found to be responsible for the transcriptional repression of cartilage-specific genes through the activation of NF-KB signaling (Hong et al., 2009; Zhong et al., 2002). HDAC4 was identified as a core element regulating hypertrophic maturation of chondrocytes and endochondral bone formation. HDAC4 not only suppresses the expression of RUNX2 at the transcriptional level via interaction with histones, but also interacts with RUNX2 directly, reducing its transcriptional activity (Vega et al., 2004). HDAC7, on the other hand, plays a role in the regulation of chondrocyte catabolism. HDAC7 activity is markedly upregulated in OA cartilage, and essentially required for IL-1 $\beta$ mediated expression of MMP13 (Higashiyama et al., 2010).

HDACs generally augment imbalance between catabolism and anabolism in chondrocytes, and the potential of HDAC inhibitors as therapeutic agents for $O A$ treatment have been extensively investigated. TSA, butyric acid (BA), and vorinostat treatment have been shown to effectively diminish IL-1mediated expression of inducible nitric oxide (NO) synthase (iNOS), COX2, and MMPs (Chabane et al., 2008; Zhong et al., 2013). These anti-inflammatory effects of HDAC inhibitors were demonstrated in vivo, with concomitant suppression of osteoarthritic cartilage destruction and synovitis (Nasu et al., 2008).

Meanwhile, histone methylation is mediated by histone methyl transferases (HMTs). Methylation can occur on both lysine and arginine residues in histones without altering the net charge of the affected residues. SOX9, a transcriptional regulator of COL2A1 and $A C A N$, is itself subjected to progressive epigenetic regulation over the course of OA progression. Under OA-related pathogenic conditions, the promoter of SOX9 acquires a epigenetic profile characterized by elevated levels of trimethylation of $\mathrm{H} 3 \mathrm{~K} 9$ and $\mathrm{H} 3 \mathrm{~K} 27$, and decreased acetylation of H3K9, $15,18,23$, and 27 , which collectively leads to transcriptional repression of SOX9 (Kim et al., 2013). Histone-lysine $\mathrm{N}$-methyltransferase SETD7 (SET7/9) forms a complex with SIRT1 in the COL2A1 promoter region and elevates trimethylation of $\mathrm{H} 3 \mathrm{~K} 4$, thereby enhancing COL2A1 expression (Oppenheimer et al., 2014). Nuclear factor of activated T-cells 1 (NFAT1) is a transcription factor whose deficiency is correlated with the onset of OA-related phenotypes (Wang et al., 2009). Agedependent expression of NFAT1 is primarily regulated by histone demethylation. Demethylation at H3K4 by lysine-specific demethylase-1A (LSD1) appears to be associated with transcriptional activation of NFAT1 during cartilage development, whereas an aging-associated increase in H3K9 methylation due to reduced lysine-specific demethylase 3A (KDM3A) level is responsible for transcriptional repression of NFAT1 (Rodova et al., 2011).

\section{EPIGENETIC EFFECTS OF OA RISK FACTORS}

\section{Aging-associated ROS}

The increasing prevalence of $O A$ in aging individuals supports the possibility that aging-associated events have causal effects on epigenetic drift, driving catabolic and anabolic imbalance in chondrocytes. Age-associated increase of oxidative stress, indicated by elevated reactive oxygen species (ROS) level, has been noted in chondrocytes (Carlo and Loeser, 2003; Jallali et al., 2005). Overall ROS level is controlled by the collective actions of enzymes such as glutathione peroxidase, superoxide dismutase, and catalase in normal chondrocytes. However, the senescence of chondrocytes disrupts this delicate balance between ROS generation and its defense mechanisms, resulting in an elevation in ROS level (Ruiz-Romero et al., 2009). An increase in ROS levels, in turn, has been shown to affect the stability and activity of histone modifying enzymes. ROS induce covalent modification of SIRT1 by carbonylating it on the thiol groups of cysteine residues between amino acid residues 467 and 492 (Caito et al., 2010). Carbonylated SIRT1 exhibits reduced protein stability, presumably becoming associated with the ubiquitin-mediated proteasome degradation pathway (Isabella Dalle - Donne, 2006). Moreover, carbonylation of SIRT1 also occurs on the $\mathrm{NAD}^{+}$binding pocket, thereby reducing its enzymatic activity (Caito et al., 2010). HDAC proteins are also a target of carbonylation under oxidative stress conditions. Oxidative stress generates 4-hydroxy-2-nonenal (4-HNE) and acrolein, which in turn react with histidine groups of HDAC2. This covalent modification in HDAC2 is responsible for reducing its protein stability (Marwick et al., 2004). Nitration also acts as a mediator, linking oxidative stress levels to HDAC2 regulation. The identified nitration sites in HDAC2 include Y167, Y173, Y146, Y68, Y73, and $\mathrm{Y} 253$, and nitration on the $\mathrm{Y} 253$ residue primes HDAC2 for the proteasome pathway (Osoata et al., 2009).

Aging-associated production of ROS elicits DNA damage, which in turn triggers activation of DNA repair mechanisms. In line with evidence indicating a role for DNA methylation in repairing processes, DNMT1 and DNMT3A were shown to be recruited to the sites of DNA damage, where they methylate neighboring CpG sites (Morano et al., 2014), potentially causing hypermethylation of the CpG sites, which results in suppression of gene expression.

\section{Metabolites and obesity}

Epigenetic modifications such as acetylation, methylation, and glycosylation are influenced by the intracellular metabolic state. By altering epigenetic profiles, cells respond to metabolic challenges and adapt to it by subsequently changing intracellular signal transduction and gene expression (Metallo and Vander Heiden, 2010).

Oxygen levels in the joint environment substantially affect energy and metabolic state of chondrocytes, influencing catabolic and anabolic factor expression (Schipani et al., 2001). In particular, in articular cartilage which is under hypoxic conditions due to its avascular nature, HIF- $1 \alpha$ may serve as an epigenetic mediator by directly inducing SIRT1 expression and activating SIRT1-driven epigenetic programs (Chen et al., 2011).

Metabolites from cellular metabolic pathways may act as sensors of energy state, and mediate corresponding epigenetic changes. Acetyl-CoA, a component of the tricarboxylic acid (TCA) cycle and an essential building block for key metabolic biomolecules, can also serve as a substrate for HATs (Kaelin and McKnight, 2013). Consequently, the intracellular acetylCoA level determines the acetylation patterns of lysine residues as a measure of metabolic state, regulating cell growth and proliferation (Cai and Tu, 2011). Moreover, the activity of ATPcitrate lyase $(A C L)$, which is an enzyme synthesizing acetylCoA from glucose-derived citrate in mammals, depends on 
Epigenetic Regulation in Osteoarthritis

Hyeonkyeong Kim et al.

Table 1. Effect of aging and metabolic abnormalities on OA progression

\begin{tabular}{|c|c|c|c|c|}
\hline Risk factors & \multicolumn{2}{|c|}{ Mediators } & Progression of OA & Reference \\
\hline $\begin{array}{l}\text { Aging } \\
\text { (Senescence) }\end{array}$ & \multicolumn{2}{|c|}{ Reactive oxygen species } & Increase & $\begin{array}{l}\text { Caito et al., 2010; Isabella Dalle - Donne, 2006; Marwick et al., 2004; } \\
\text { Osoata et al., } 2009\end{array}$ \\
\hline \multirow[t]{6}{*}{ Obesity } & \multirow{2}{*}{\multicolumn{2}{|c|}{ Mechanical loading }} & Increase & Guilak 2011; Maly et al., 2005; Mundermann et al., 2005 \\
\hline & & & No effect & Carman et al., 1994 \\
\hline & \multirow[t]{4}{*}{ Adipokine } & Leptin & Increase & Iliopoulos et al., 2007; Otero et al., 2005 \\
\hline & & Adiponectin & Increase & Kang et al., 2010 \\
\hline & & & Decrease & Chen et al., 2006; Yusuf et al., 2011 \\
\hline & & Visfatin & Increase & $\begin{array}{l}\text { Duan Hao et al., 2012; Gosset et al., 2008; Yammani and Loeser, 2012; } \\
\text { Yang et al., } 2015\end{array}$ \\
\hline Hypertension & \multicolumn{2}{|c|}{ Subchondral ischemia } & Increase & Hong et al., 2011 \\
\hline
\end{tabular}

glucose abundance. Therefore, ACL is capable of serving as a nutrient sensor, linking intracellular energy state into histone acetylation and gene expression profiles (Hatzivassiliou et al., 2005; Wellen et al., 2009).

Methyltransferases use S-adenosylmethionine (SAM), derived from the methionine cycle, as a methyl group donor. The production rate of SAM is regulated by the activity of ATPdependent methionine adenosyltransferase (MAT). Thus, cellular ATP controls the intracellular level of SAM and the activity of various methyltransferases, in turn influencing transcription profiles (Nishikawa et al., 2015). Many other enzymes regulating epigenetic changes use biological metabolites such as $\mathrm{NAD}^{+}$(protein deacetylase/mono-ADP ribosyltransferase, and sirtuin), FAD (FAD-dependent histone demethylase), and 2oxoglutarate (2-oxoglutarate-dependent histone demethylase) as cofactors, or UDP-N-acetylglucosamine (O-linked N-acetylglucosamine transferase) as a substrate.

The effect of metabolites on epigenetic regulation supports the link between metabolism and the molecular pathogenesis of various diseases (Kaelin and McKnight, 2013). SIRT family members have been demonstrated to be key metabolic regulators in controlling glucose and lipid metabolism (Picard et al., 2004; Schwer and Verdin, 2008), and gluconeogenesis in muscle and liver (Liu et al., 2008). SIRT1 promotes insulin secretion and ATP production upon glucose stimulation of pancreatic $\beta$ cells (Bordone et al., 2006). In metabolic syndrome patients with insulin resistance or atherosclerosis, downregulation of SIRT1 was observed in monocytes in response to glucose or fatty acid treatment (de Kreutzenberg et al., 2010). Isoforms of the HDAC family are implicated in regulating lipid metabolism during adipogenesis (lyer et al., 2012). Genetic deletion of Hdac1 and Hdac2 in mesenchymal precursor cells results in reduced adipogenesis and adipocyte differentiation, (Haberland et al., 2010), while Hdac9 knockout mice exhibited accelerated adipogenic differentiation (Chatterjee et al., 2011). Protein demethylase KDM3A is coupled to the regulation of genes involved in beta-adrenergic stimulated glycerol release and fatty acid oxidation such as uncoupling protein 1 (UCP1) (Tateishi et al., 2009). Extensive correlation between epigenetics and abnormal metabolic state have been noted in metabolic diseases such as obesity (Campion et al., 2009) and type 2 diabetes (Campion et al., 2009; Ling and Groop, 2009).

There has been substantial evidence for the idea that the prevalence of OA is positively correlated with that of metabolic syndrome (Table 1) (Kornaat et al., 2009; Puenpatom and Victor, 2009; Zhuo et al., 2012). Among the main features of metabolic syndrome, obesity has been considered to be a major risk factor for OA occurrence in the joints of knees, hip, and hands (Griffin and Guilak, 2005; Oliveria et al., 1999). However, a mechanistic understanding of the association between obesity and OA remains elusive. The simplest view indicates that obesity exacerbates joint strength by imposing a higher degree of mechanical loading on the joint (Guilak, 2011). Clinical studies have supported the idea that abnormal loading jeopardizes the structure and integrity of the knee joint, facilitating OA development (Maly et al., 2005; Mundermann et al., 2005). However, the notion that mechanical loading is the sole factor for obesity-induced OA is controversial. A series of studies demonstrate that the incidence and severity of hand OA have a positive correlation with body mass index (BMI), although mechanical loading from body weight cannot participate in the pathogenesis of this type of OA (Carman et al., 1994).

Adipokines released from fat tissues may represent a potential link between obesity and OA. Strikingly, obese mice with impairment in leptin signaling, due to deletion of leptin or leptin receptor, do not exhibit differences in terms of severity of OA in comparison to mice with normal body weight (Griffin et al., 2009). In fact, synovial fluid of OA patients was characterized to have elevated levels of leptin (Dumond et al., 2003; Ku et al., 2009). Increased levels of leptin in synovial fluid and cartilage promote cartilage catabolism by upregulating MMP13 and NOS2 expression (Iliopoulos et al., 2007; Otero et al., 2005).

Other adipokines, such as adiponectin (Chen et al., 2006; Lago et al., 2008) and visfatin, (Duan et al., 2012) were found to be responsible for the regulation of chondrocyte metabolism. Adiponectin is known to regulate homeostasis of whole body energy by controlling glucose and lipid metabolism, and abnormalities in its regulation have been implicated in several metabolic disorders including obesity (Kim et al., 2015). While alterations of adiponectin level were noted in OA cartilage and synovial fluid (Honsawek and Chayanupatkul, 2010; Koskinen et al., 2011; Yusuf et al., 2011), the role of this adipokine in OA development remains controversial. A study by Kang and colleagues suggests that that adiponectin promotes chondrocyte catabolism by upregulating MMPs and NOS2 levels via AMPK and JNK pathways (Kang et al., 2010). Others have suggested a chondroprotective function for adiponectin, demonstrating its role in upregulating inhibition of metalloproteinase 2 (TIMP2) and downregulating MMP13 (Chen et al., 2006; Yusuf et al., 2011). Visfatin (encoded by NAMPT) is an adipokine that has been found to be upregulated in chondrocytes, synovial fluid, and synoviocytes on OA. Visfatin inhibits matrix proteoglycan 


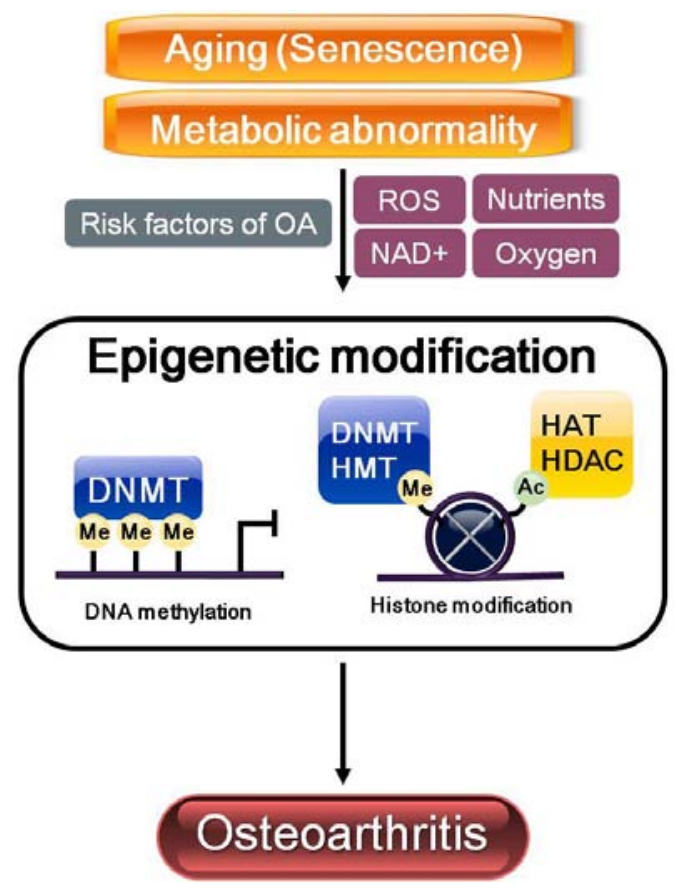

Fig. 2. Overview of epigenetic regulation in OA pathogenesis. OAassociated risk factors such as aging (senescence) and metabolic abnormalities drive epigenetic shift at the level of DNA methylation and histone modification. These epigenetic changes elicit an imbalance between chondrocyte catabolism and anabolism, leading to OA development.

synthesis and increases the expression of matrix-degradative enzymes (Duan et al., 2012; Gosset et al., 2008; Yammani and Loeser, 2012; Yang et al., 2015). Notably, visfatin can be localized intracellularly, serving as a regulator of salvage pathways of $\mathrm{NAD}^{+}$synthesis. Increased level of $\mathrm{NAD}^{+}$by intracellular visfatin enhances enzymatic activity of SIRT1, affecting the expression of SOX9 and type II collagen through epigenetic regulation (Hong et al., 2011).

In addition to obese individuals, patients with hypertension are highly likely to suffer from OA (Conaghan et al., 2005; Hart et al., 1995). Hypertension triggers the onset of subchondral ischemia, which is considered to promote the pathogenesis of $\mathrm{OA}$, by restricting the formation of subchondral vessels and limiting nutrient and gas support to cartilage (Findlay, 2007). Hypertension is presumed to affect a subset of epigenetic pathways that are regulated by glucose or oxygen in cartilage.

\section{CONCLUSION}

The recognition of non-genetic factors, such as aging or obesity, as principal risk factors for OA has highlighted the potential significance of epigenetics in the pathogenesis of this disease (Fig. 2). A series of studies reviewed in this paper indicate that various types of epigenetic drift at the level of DNA methylation or histone modification are implicated in regulating chondrocyte catabolism and anabolism, over the course of OA development. These epigenetic regulations in chondrocytes are substantially affected by cellular senescence, or energy and metabolic state. Moreover, the close correlation between the occurrence of OA and metabolic disorders supports the idea that OA is a metabolic disease. Animal studies targeting the components of epigenetic regulators have demonstrated the potential of these compounds as therapeutic options for OA treatment. Rapid advances in molecular biology techniques, such as epigenome sequencing and ChIP technologies, are expected to lead to a more comprehensive understanding of epigenetic regulation in the pathogenesis of $O A$.

\section{ACKNOWLEDGMENTS}

This work was supported by grants from Bio \& Medical Technology Development Program of the National Research Foundation funded by the Korean government (NRF-2015M 3A9E6028674), the National R\&D Program for Cancer Control, Ministry for Health and Welfare, Republic of Korea (1520070), and Research Resettlement Fund for the new faculty of Seoul National University.

\section{REFERENCES}

Blander, G., and Guarente, L. (2004). The Sir2 family of protein deacetylases. Ann. Rev. Biochem. 73, 417-435.

Bordone, L., Motta, M.C., Picard, F., Robinson, A., Jhala, U.S., Apfeld, J., McDonagh, T., Lemieux, M., McBurney, M., Szilvasi, A., et al. (2006). Sirt1 regulates insulin secretion by repressing UCP2 in pancreatic beta cells. PLoS Biol. 4, e31.

Buckwalter, J.A., and Brown, T.D. (2004). Joint injury, repair, and remodeling: roles in post-traumatic osteoarthritis. Clin. Orthop. Relat. Res. 2004, 7-16.

Bui, C., Barter, M.J., Scott, J.L., Xu, Y., Galler, M., Reynard, L.N., Rowan, A.D., and Young, D.A. (2012). cAMP response elementbinding (CREB) recruitment following a specific CpG demethylation leads to the elevated expression of the matrix metalloproteinase 13 in human articular chondrocytes and osteoarthritis. FASEB J. 26, 3000-3011.

Cai, L., and Tu, B.P. (2011). On acetyl-CoA as a gauge of cellular metabolic state. Cold Spring Harb. Symp. Quant. Biol. 76, 195202.

Caito, S., Rajendrasozhan, S., Cook, S., Chung, S., Yao, H., Friedman, A.E., Brookes, P.S., and Rahman, I. (2010). SIRT1 is a redox-sensitive deacetylase that is post-translationally modified by oxidants and carbonyl stress. FASEB J. 24, 31453159.

Campion, J., Milagro, F.I., and Martinez, J.A. (2009). Individuality and epigenetics in obesity. Obesity Rev. 10, 383-392.

Carlo, M.D., Jr., and Loeser, R.F. (2003). Increased oxidative stress with aging reduces chondrocyte survival: correlation with intracellular glutathione levels. Arthritis. Rheum. 48, 3419-3430.

Carman, W.J., Sowers, M., Hawthorne, V.M., and Weissfeld, L.A. (1994). Obesity as a risk factor for osteoarthritis of the hand and wrist: a prospective study. Am. J. Epidemiol. 139, 119-129.

Chabane, N., Zayed, N., Afif, H., Mfuna-Endam, L., Benderdour, M. Boileau, C., Martel-Pelletier, J., Pelletier, J.P., Duval, N., and Fahmi, H. (2008). Histone deacetylase inhibitors suppress interleukin-1beta-induced nitric oxide and prostaglandin E2 production in human chondrocytes. Osteoarthritis Cartilage 16, 1267-1274.

Chatterjee, T.K., Idelman, G., Blanco, V., Blomkalns, A.L., Piegore, M.G., Jr., Weintraub, D.S., Kumar, S., Rajsheker, S., Manka, D., Rudich, S.M., et al. (2011). Histone deacetylase 9 is a negative regulator of adipogenic differentiation. J. Biol. Chem. 286, 27836-27847.

Chen, T.H., Chen, L., Hsieh, M.S., Chang, C.P., Chou, D.T., and Tsai, S.H. (2006). Evidence for a protective role for adiponectin in osteoarthritis. Biochim. Biophys. Acta 1762, 711-718.

Chen, R., Dioum, E.M., Hogg, R.T., Gerard, R.D., and Garcia, J.A. (2011). Hypoxia increases sirtuin 1 expression in a hypoxiainducible factor-dependent manner. J. Biol. Chem. 286, 1386913878.

Conaghan, P.G., Vanharanta, H., and Dieppe, P.A. (2005). Is progressive osteoarthritis an atheromatous vascular disease? Ann. Rheum. Dis. 64, 1539-1541. 
de Kreutzenberg, S.V., Ceolotto, G., Papparella, I., Bortoluzzi, A., Semplicini, A., Dalla Man, C., Cobelli, C., Fadini, G.P., and Avogaro, A. (2010). Downregulation of the longevity-associated protein sirtuin 1 in insulin resistance and metabolic syndrome: potential biochemical mechanisms. Diabetes 59, 1006-1015.

de Ruijter, A.J., van Gennip, A.H., Caron, H.N., Kemp, S., and van Kuilenburg, A.B. (2003). Histone deacetylases (HDACs): characterization of the classical HDAC family. Biochem. J. 370, 737-749.

Dillon, C.F., Rasch, E.K., Gu, Q., and Hirsch, R. (2006). Prevalence of knee osteoarthritis in the United States: arthritis data from the Third National Health and Nutrition Examination Survey 1991-94. J. Rheumatol. 33, 2271-2279.

Duan, Y., Hao, D., Li, M., Wu, Z., Li, D., Yang, X., and Qiu, G. (2012) Increased synovial fluid visfatin is positively linked to cartilage degradation biomarkers in osteoarthritis. Rheumatol. Int. 32, 985-990.

Dumond, H., Presle, N., Terlain, B., Mainard, D., Loeuille, D., Netter, P., and Pottie, P. (2003). Evidence for a key role of leptin in osteoarthritis. Arthritis Rheum. 48, 3118-3129.

Dvir-Ginzberg, M., Gagarina, V., Lee, E.J., and Hall, D.J. (2008). Regulation of cartilage-specific gene expression in human chondrocytes by SirT1 and nicotinamide phosphoribosyltransferase. J. Biol. Chem. 283, 36300-36310.

Dvir-Ginzberg, M., Gagarina, V., Lee, E.J., Booth, R., Gabay, O., and Hall, D.J. (2011). Tumor necrosis factor alpha-mediated cleavage and inactivation of SirT1 in human osteoarthritic chondrocytes. Arthritis Rheum. 63, 2363-2373.

Fernandez-Tajes, J., Soto-Hermida, A., Vazquez-Mosquera, M.E. Cortes-Pereira, E., Mosquera, A., Fernandez-Moreno, M., Oreiro, N., Fernandez-Lopez, C., Fernandez, J.L., Rego-Perez, I., et al. (2014). Genome-wide DNA methylation analysis of articular chondrocytes reveals a cluster of osteoarthritic patients. Ann. Rheum. Dis. 73, 668-677.

Findlay, D.M. (2007). Vascular pathology and osteoarthritis. Rheumatology 46, 1763-1768.

Fujita, N., Matsushita, T., Ishida, K., Kubo, S., Matsumoto, T., Takayama, K., Kurosaka, M., and Kuroda, R. (2011). Potential involvement of SIRT1 in the pathogenesis of osteoarthritis through the modulation of chondrocyte gene expressions. J. Orthop. Res. 29, 511-515.

Furumatsu, T., and Asahara, H. (2010). Histone acetylation influences the activity of Sox9-related transcriptional complex. Acta Med. Okayama 64, 351-357.

Furumatsu, T., Tsuda, M., Yoshida, K., Taniguchi, N., Ito, T., Hashimoto, M., Ito, T., and Asahara, H. (2005). Sox9 and p300 cooperatively regulate chromatin-mediated transcription. J. Biol. Chem. 280, 35203-35208.

Gabay, O., Oppenhiemer, H., Meir, H., Zaal, K., Sanchez, C., and Dvir-Ginzberg, M. (2012). Increased apoptotic chondrocytes in articular cartilage from adult heterozygous SirT1 mice. Ann. Rheum. Dis. 71, 613-616.

Gabay, O., Sanchez, C., Dvir-Ginzberg, M., Gagarina, V., Zaal, K.J., Song, Y., He, X.H., and McBurney, M.W. (2013). Sirtuin 1 enzymatic activity is required for cartilage homeostasis in vivo in a mouse model. Arthritis Rheum. 65, 159-166.

Gagarina, V., Gabay, O., Dvir-Ginzberg, M., Lee, E.J., Brady, J.K., Quon, M.J., and Hall, D.J. (2010). SirT1 enhances survival of human osteoarthritic chondrocytes by repressing protein tyrosine phosphatase 1B and activating the insulin-like growth factor receptor pathway. Arthritis Rheum. 62, 1383-1392.

Gosset, M., Berenbaum, F., Salvat, C., Sautet, A., Pigenet, A., Tahiri, K., and Jacques, C. (2008). Crucial role of visfatin/pre-B cell colony-enhancing factor in matrix degradation and prostaglandin E2 synthesis in chondrocytes: possible influence on osteoarthritis. Arthritis Rheum. 58, 1399-1409.

Griffin, T.M., and Guilak, F. (2005). The role of mechanical loading in the onset and progression of osteoarthritis. Exerc. Sport Sci. Rev. 33, 195-200.

Griffin, T.M., Huebner, J.L., Kraus, V.B., and Guilak, F. (2009). Extreme obesity due to impaired leptin signaling in mice does not cause knee osteoarthritis. Arthritis Rheum. 60, 2935-2944.

Guilak, F. (2011). Biomechanical factors in osteoarthritis. Best practice \& research. Clin. Rheumatol. 25, 815-823.

Haberland, M., Carrer, M., Mokalled, M.H., Montgomery, R.L., and Olson, E.N. (2010). Redundant control of adipogenesis by histone deacetylases 1 and 2. J. Biol. Chem. 285, 14663-14670. Hart, D.J., Doyle, D.V., and Spector, T.D. (1995). Association between metabolic factors and knee osteoarthritis in women: the Chingford Study. J. Rheumatol. 22, 1118-1123.

Hashimoto, K., Oreffo, R.O., Gibson, M.B., Goldring, M.B., and Roach, H.I. (2009). DNA demethylation at specific CpG sites in the IL1B promoter in response to inflammatory cytokines in human articular chondrocytes. Arthritis Rheum. 60, 3303-3313.

Hashimoto, K., Otero, M., Imagawa, K., de Andres, M.C., Coico, J.M., Roach, H.I., Oreffo, R.O., Marcu, K.B., and Goldring, M.B. (2013). Regulated transcription of human matrix metalloproteinase 13 (MMP13) and interleukin-1beta (IL1B) genes in chondrocytes depends on methylation of specific proximal promoter CpG sites. J. Biol. Chem. 288, 10061-10072.

Hatzivassiliou, G., Zhao, F., Bauer, D.E., Andreadis, C., Shaw, A.N., Dhanak, D., Hingorani, S.R., Tuveson, D.A., and Thompson, C.B. (2005). ATP citrate lyase inhibition can suppress tumor cell growth. Cancer Cell 8, 311-321.

Heinegard, D., and Saxne, T. (2011). The role of the cartilage matrix in osteoarthritis. Nat. Rev. Rheumatol. 7, 50-56.

Higashiyama, R., Miyaki, S., Yamashita, S., Yoshitaka, T., Lindman, G., Ito, Y., Sasho, T., Takahashi, K., Lotz, M., and Asahara, H. (2010). Correlation between MMP-13 and HDAC7 expression in human knee osteoarthritis. Mod. Rheumatol. 20, 11-17.

Hong, S., Derfoul, A., Pereira-Mouries, L., and Hall, D.J. (2009). A novel domain in histone deacetylase 1 and 2 mediates repression of cartilage-specific genes in human chondrocytes. FASEB J. 23, 3539-3552.

Hong, E.H., Yun, H.S., Kim, J., Um, H.D., Lee, K.H., Kang, C.M., Lee, S.J., Chun, J.S., and Hwang, S.G. (2011). Nicotinamide phosphoribosyltransferase is essential for interleukin-1betamediated dedifferentiation of articular chondrocytes via SIRT1 and extracellular signal-regulated kinase (ERK) complex signaling. J. Biol. Chem. 286, 28619-28631.

Honsawek, S., and Chayanupatkul, M. (2010). Correlation of plasma and synovial fluid adiponectin with knee osteoarthritis severity. Arch. Med. Res. 41, 593-598.

Houard, X., Goldring, M.B., and Berenbaum, F. (2013). Homeostatic mechanisms in articular cartilage and role of inflammation in osteoarthritis. Curr. Rheumatol. Rep. 15, 375.

Huh, Y.H., Ryu, J.H., and Chun, J.S. (2007). Regulation of type II collagen expression by histone deacetylase in articular chondrocytes. J. Biol. Chem. 282, 17123-17131.

lliopoulos, D., Malizos, K.N., and Tsezou, A. (2007). Epigenetic regulation of leptin affects MMP-13 expression in osteoarthritic chondrocytes: possible molecular target for osteoarthritis therapeutic intervention. Ann. Rheumatic Dis. 66, 1616-1621.

Imagawa, K., de Andres, M.C., Hashimoto, K., Itoi, E., Otero, M. Roach, H.I., Goldring, M.B., and Oreffo, R.O. (2014). Association of reduced type IX collagen gene expression in human osteoarthritic chondrocytes with epigenetic silencing by DNA hypermethylation. Arthritis Rheumatol. 66, 3040-3051.

Isabella Dalle - Donne, G.A., Marina Carini, Roberto Colombo, Ranieri Rossi, Aldo Milzani (2006). Protein carbonylation, cellular dysfunction, and disease progre. J. Cell. Mol. Med. 10, 389-406.

Iyer, A., Fairlie, D.P., and Brown, L. (2012). Lysine acetylation in obesity, diabetes and metabolic disease. Immunol. Cell Biol. 90, 39-46.

Jallali, N., Ridha, H., Thrasivoulou, C., Underwood, C., Butler, P.E., and Cowen, T. (2005). Vulnerability to ROS-induced cell death in ageing articular cartilage: the role of antioxidant enzyme activity. Osteoarthritis Cartilage 13, 614-622.

Kaelin, W.G., Jr., and McKnight, S.L. (2013). Influence of metabolism on epigenetics and disease. Cell 153, 56-69.

Kang, E.H., Lee, Y.J., Kim, T.K., Chang, C.B., Chung, J.H., Shin, K., Lee, E.Y., Lee, E.B., and Song, Y.W. (2010). Adiponectin is a potential catabolic mediator in osteoarthritis cartilage. Arthritis Res. Ther. 12, R231.

Kim, K.I., Park, Y.S., and Im, G.I. (2013). Changes in the epigenetic status of the SOX-9 promoter in human osteoarthritic cartilage. J. Bone Miner. Res. 28, 1050-1060

Kim, J.H., Jeon, J., Shin, M., Won, Y., Lee, M., Kwak, J.S., Lee, G., Rhee, J., Ryu, J.H., Chun, C.H., et al. (2014). Regulation of the catabolic cascade in osteoarthritis by the zinc-ZIP8-MTF1 axis. Cell $156,730-743$ 
Kim, A.Y., Park, Y.J., Pan, X., Shin, K.C., Kwak, S.H., Bassas, A.F., Sallam, R.M., Park, K.S., Alfadda, A.A., Xu, A., et al. (2015). Obesity-induced DNA hypermethylation of the adiponectin gene mediates insulin resistance. Nat. Commun. 6, 7585.

Knudson, C.B., and Knudson, W. (2001). Cartilage proteoglycans. Semin. Cell Dev. Biol. 12, 69-78.

Kornaat, P.R., Sharma, R., van der Geest, R.J., Lamb, H.J., Kloppenburg, M., Hellio le Graverand, M.P., Bloem, J.L., and Watt, I. (2009). Positive association between increased popliteal artery vessel wall thickness and generalized osteoarthritis: is OA also part of the metabolic syndrome? Skeletal Radiol. 38, 11471151.

Koskinen, A., Juslin, S., Nieminen, R., Moilanen, T., Vuolteenaho, K., and Moilanen, E. (2011). Adiponectin associates with markers of cartilage degradation in osteoarthritis and induces production of proinflammatory and catabolic factors through mitogen-activated protein kinase pathways. Arthritis Res. Ther. 13, R184

Kouzarides, T. (2007). Chromatin modifications and their function. Cell 128, 693-705.

Ku, J.H., Lee, C.K., Joo, B.S., An, B.M., Choi, S.H., Wang, T.H., and Cho, H.L. (2009). Correlation of synovial fluid leptin concentrations with the severity of osteoarthritis. Clin. Rheumatol. 28, 1431-1435.

Lago, R., Gomez, R., Otero, M., Lago, F., Gallego, R., Dieguez, C., Gomez-Reino, J.J., and Gualillo, O. (2008). A new player in cartilage homeostasis: adiponectin induces nitric oxide synthase type II and pro-inflammatory cytokines in chondrocytes. Osteoarthritis Cartilage 16, 1101-1109.

Lawrence, R.C., Felson, D.T., Helmick, C.G., Arnold, L.M., Choi, H., Deyo, R.A., Gabriel, S., Hirsch, R., Hochberg, M.C., Hunder, G.G., et al. (2008). Estimates of the prevalence of arthritis and other rheumatic conditions in the United States. Part II. Arthritis Rheum. 58, 26-35.

Lefebvre, V., Li, P., and de Crombrugghe, B. (1998). A new long form of Sox5 (L-Sox5), Sox6 and Sox9 are coexpressed in chondrogenesis and cooperatively activate the type II collagen gene. EMBO J. 17, 5718-5733.

Ling, C., and Groop, L. (2009). Epigenetics: a molecular link between environmental factors and type 2 diabetes. Diabetes 58 2718-2725.

Liu, Y., Dentin, R., Chen, D., Hedrick, S., Ravnskjaer, K., Schenk, S., Milne, J., Meyers, D.J., Cole, P., Yates, J., 3rd, et al. (2008). A fasting inducible switch modulates gluconeogenesis via activator/coactivator exchange. Nature 456, 269-273.

Loeser, R.F. (2013). Aging processes and the development of osteoarthritis. Curr. Opin. Rheumatol. 25, 108-113.

Maly, M.R., Costigan, P.A., and Olney, S.J. (2005). Contribution of psychosocial and mechanical variables to physical performance measures in knee osteoarthritis. Phys. Ther. 85, 1318-1328.

Marwick, J.A., Kirkham, P.A., Stevenson, C.S., Danahay, H., Giddings, J. Butler, K. Donaldson, K. Macnee, W. and Rahman, I. (2004). Cigarette smoke alters chromatin remodeling and induces proinflammatory genes in rat lungs. Am. J. Respir. Cell Mol. Biol. 31, 633-642.

Matsuzaki, T., Matsushita, T., Takayama, K., Matsumoto, T., Nishida, K., Kuroda, R., and Kurosaka, M. (2014). Disruption of Sirt1 in chondrocytes causes accelerated progression of osteoarthritis under mechanical stress and during ageing in mice. Ann. Rheumatic Dis. 73, 1397-1404.

Metallo, C.M., and Vander Heiden, M.G. (2010). Metabolism strikes back: metabolic flux regulates cell signaling. Genes Dev. 24, 2717-2722.

Morano, A., Angrisano, T., Russo, G., Landi, R., Pezone, A., Bartollino, S., Zuchegna, C., Babbio, F., Bonapace, I.M., Allen, B., et al. (2014). Targeted DNA methylation by homologydirected repair in mammalian cells. Transcription reshapes methylation on the repaired gene. Nucleic Acids Res. 42, 804821.

Mundermann, A., Dyrby, C.O., and Andriacchi, T.P. (2005). Secondary gait changes in patients with medial compartment knee osteoarthritis: increased load at the ankle, knee, and hip during walking. Arthritis Rheum. 52, 2835-2844.

Nagai, K., Matsushita, T., Matsuzaki, T., Takayama, K., Matsumoto, T., Kuroda, R., and Kurosaka, M. (2015). Depletion of SIRT6 causes cellular senescence, DNA damage, and telomere dysfunction in human chondrocytes. Osteoarthritis Cartilage 23,
$1412-1420$

Nasu, Y., Nishida, K., Miyazawa, S., Komiyama, T., Kadota, Y., Abe N., Yoshida, A., Hirohata, S., Ohtsuka, A., and Ozaki, T. (2008), Trichostatin A, a histone deacetylase inhibitor, suppresses synovial inflammation and subsequent cartilage destruction in a collagen antibody-induced arthritis mouse model. Osteoarthritis Cartilage 16, 723-732.

Nishikawa, K., Iwamoto, Y., Kobayashi, Y., Katsuoka, F., Kawaguchi, S., Tsujita, T., Nakamura, T., Kato, S., Yamamoto, M., Takayanagi, $H_{\text {., }}$ et al. (2015). DNA methyltransferase 3a regulates osteoclast differentiation by coupling to an $\mathrm{S}$ adenosylmethionine-producing metabolic pathway. Nat. Med. 21 , 281-287.

Oliveria, S.A., Felson, D.T., Cirillo, P.A., Reed, J.I., and Walker, A.M. (1999). Body weight, body mass index, and incident symptomatic osteoarthritis of the hand, hip, and knee. Epidemiology 10, 161166

Oppenheimer, H., Gabay, O., Meir, H., Haze, A., Kandel, L., Liebergall, M., Gagarina, V., Lee, E.J., and Dvir-Ginzberg, M (2012). 75-kd sirtuin 1 blocks tumor necrosis factor alphamediated apoptosis in human osteoarthritic chondrocytes. Arthritis Rheum. 64, 718-728.

Oppenheimer, H., Kumar, A., Meir, H., Schwartz, I., Zini, A., Haze, A., Kandel, L., Mattan, Y., Liebergall, M., and Dvir-Ginzberg, M. (2014). Set7/9 impacts COL2A1 expression through binding and repression of SirT1 histone deacetylation. J. Bone Miner. Res. 29, 348-360.

Osoata, G.O., Yamamura, S., Ito, M., Vuppusetty, C., Adcock, I.M., Barnes, P.J., and Ito, K. (2009). Nitration of distinct tyrosine residues causes inactivation of histone deacetylase 2 . Biochem. Biophys. Res. Commun. 384, 366-371.

Otero, M., Lago, R., Lago, F., Reino, J.J., and Gualillo, O. (2005) Signalling pathway involved in nitric oxide synthase type II activation in chondrocytes: synergistic effect of leptin with interleukin-1. Arthritis Res. Ther. 7, R581-591.

Picard, F., Kurtev, M., Chung, N., Topark-Ngarm, A., Senawong, T., Machado De Oliveira, R., Leid, M., McBurney, M.W., and Guarente, L. (2004). Sirt1 promotes fat mobilization in white adipocytes by repressing PPAR-gamma. Nature 429, 771-776.

Poschl, E., Fidler, A., Schmidt, B., Kallipolitou, A., Schmid, E., and Aigner, T. (2005). DNA methylation is not likely to be responsible for aggrecan down regulation in aged or osteoarthritic cartilage. Ann. Rheumatic Dis. 64, 477-480.

Pradhan, S., and Esteve, P.-O. (2003). Mammalian DNA (cytosine5) methyltransferases and their expression. Clin. Immunol. 109, 6-16.

Puenpatom, R.A., and Victor, T.W. (2009). Increased prevalence of metabolic syndrome in individuals with osteoarthritis: an analysis of NHANES III data. Postgrad. Med. 121, 9-20.

Razin, A. (1998). CpG methylation, chromatin structure and gene silencing-a three-way connection. EMBO J. 17, 4905-4908.

Reynard, L.N., Bui, C., Canty-Laird, E.G., Young, D.A., and Loughlin J. (2011). Expression of the osteoarthritis-associated gene GDF5 is modulated epigenetically by DNA methylation. Hum. Mol. Genet. 20, 3450-3460.

Roach, H.I., Yamada, N., Cheung, K.S., Tilley, S., Clarke, N.M., Oreffo, R.O., Kokubun, S., and Bronner, F. (2005). Association between the abnormal expression of matrix-degrading enzymes by human osteoarthritic chondrocytes and demethylation of specific CpG sites in the promoter regions. Arthritis Rheum. 52, 3110-3124

Rodova, M., Lu, Q., Li, Y., Woodbury, B.G., Crist, J.D., Gardner, B.M., Yost, J.G., Zhong, X.B., Anderson, H.C., and Wang, J. (2011). Nfat1 regulates adult articular chondrocyte function through its age-dependent expression mediated by epigenetic histone methylation. J. Bone Miner. Res. 26, 1974-1986.

Ruiz-Romero, C., Calamia, V., Mateos, J.s., Carreira, V., MartínezGomariz, M., Fernández, M.F., and Blanco, F.J. (2009). Mitochondrial dysregulation of osteoarthritic human articular chondrocytes analyzed by proteomics a decrease in mitochondrial superoxide dismutase points to a redox imbalance. Mol. Cell. Proteomics 8, 172-189.

Saito, T., Fukai, A., Mabuchi, A., Ikeda, T., Yano, F., Ohba, S., Nishida, N., Akune, T., Yoshimura, N., Nakagawa, T., et al. (2010). Transcriptional regulation of endochondral ossification by HIF-2alpha during skeletal growth and osteoarthritis develop- 
ment. Nat. Med. 16, 678-686.

Schipani, E., Ryan, H.E., Didrickson, S., Kobayashi, T., Knight, M. and Johnson, R.S. (2001). Hypoxia in cartilage: HIF-1alpha is essential for chondrocyte growth arrest and survival. Genes Dev. $15,2865-2876$

Schwer, B., and Verdin, E. (2008). Conserved metabolic regulatory functions of sirtuins. Cell Metab. 7, 104-112.

Sesselmann, S., Söder, S., Voigt, R., Haag, J., Grogan, S., and Aigner, T. (2009). DNA methylation is not responsible for p21WAF1/CIP1 down-regulation in osteoarthritic chondrocytes. Osteoarthritis Cartilage 17, 507-512.

Sterner, D.E., and Berger, S.L. (2000). Acetylation of histones and transcription-related factors. Microbiol. Mol. Biol. Rev. 64, 435459

Tateishi, K., Okada, Y., Kallin, E.M., and Zhang, Y. (2009). Role of Jhdm2a in regulating metabolic gene expression and obesity resistance. Nature 458, 757-761.

Troeberg, L., and Nagase, H. (2012). Proteases involved in cartilage matrix degradation in osteoarthritis. Biochim. Biophys. Acta 1824, 133-145.

Vega, R.B., Matsuda, K., Oh, J., Barbosa, A.C., Yang, X., Meadows, E., McAnally, J., Pomajzl, C., Shelton, J.M., Richardson, J.A., et al. (2004). Histone deacetylase 4 controls chondrocyte hypertrophy during skeletogenesis. Cell 119, 555-566.

Wang, J., Gardner, B.M., Lu, Q., Rodova, M., Woodbury, B.G., Yost, J.G., Roby, K.F., Pinson, D.M., Tawfik, O., and Anderson, H.C. (2009). Transcription factor Nfat1 deficiency causes osteoarthritis through dysfunction of adult articular chondrocytes. J. Pathol. 219, 163-172.

Wellen, K.E., Hatzivassiliou, G., Sachdeva, U.M., Bui, T.V., Cross, J.R., and Thompson, C.B. (2009). ATP-citrate lyase links cellular metabolism to histone acetylation. Science 324, 1076-1080.

Wluka, A.E., Lombard, C.B., and Cicuttini, F.M. (2013). Tackling obesity in knee osteoarthritis. Nat. Rev. Rheumatol. 9, 225-235.
Yammani, R.R., and Loeser, R.F. (2012). Extracellular nicotinamide phosphoribosyltransferase (NAMPT/visfatin) inhibits insulin-like growth factor-1 signaling and proteoglycan synthesis in human articular chondrocytes. Arthritis Res. Ther. 14, R23.

Yang, S., Kim, J., Ryu, J.H., Oh, H., Chun, C.H., Kim, B.J., Min, B.H., and Chun, J.S. (2010). Hypoxia-inducible factor-2alpha is a catabolic regulator of osteoarthritic cartilage destruction. Nat. Med. 16, 687-693.

Yang, S., Ryu, J.H., Oh, H., Jeon, J., Kwak, J.S., Kim, J.H., Kim H.A., Chun, C.H., and Chun, J.S. (2015). NAMPT (visfatin), a direct target of hypoxia-inducible factor-2alpha, is an essentia catabolic regulator of osteoarthritis. Ann. Rheumatic Dis. 74, 595-602.

Yusuf, E., Ioan-Facsinay, A., Bijsterbosch, J., Klein-Wieringa, I., Kwekkeboom, J., Slagboom, P.E., Huizinga, T.W., and Kloppenburg, M. (2011). Association between leptin, adiponectin and resistin and long-term progression of hand osteoarthritis. Ann. Rheumatic Dis. 70, 1282-1284.

Zhong, H., May, M.J., Jimi, E., and Ghosh, S. (2002). The phosphorylation status of nuclear NF-kappa B determines its association with CBP/p300 or HDAC-1. Mol. Cell 9, 625-636.

Zhong, H.M., Ding, Q.H., Chen, W.P., and Luo, R.B. (2013) Vorinostat, a HDAC inhibitor, showed anti-osteoarthritic activities through inhibition of iNOS and MMP expression, p38 and ERK phosphorylation and blocking NF-kappaB nuclear translocation. Int. Immunopharmacol. 17, 329-335.

Zhuo, Q., Yang, W., Chen, J., and Wang, Y. (2012). Metabolic syndrome meets osteoarthritis. Nat. Rev. Rheumatol. 8, 729-737.

Zimmermann, P., Boeuf, S., Dickhut, A., Boehmer, S., Olek, S., and Richter, W. (2008). Correlation of COL10A1 induction during chondrogenesis of mesenchymal stem cells with demethylation of two CpG sites in the COL10A1 promoter. Arthritis Rheum. 58, 2743-2753. 